\title{
Zur diagnostischen Relevanz von CBCL und YSR bei Diagnose einer PTBS im Jugendalter
}

\author{
Sabine Völkl-Kernstock · Katrin Skala · Sophie Klomfar • Oswald D. Kothgassner
}

Eingegangen: 10. August 2016 / Angenommen: 22. Oktober 2016 / Online publiziert: 9. November 2016 (c) Der/die Autor(en) 2016. Dieser Artikel ist eine Open-Access-Publikation.

\section{Zusammenfassung}

Grundlagen In der vorliegenden Studie wird die Symptomatik von Kindern und Jugendlichen, die traumatisierende Erfahrungen erlebten, mittels der deutschen Form der Child Behavior Checklist (CBCL) und des Youth Self Reports (YSR) untersucht. Dabei soll der Frage nachgegangen werden, inwiefern sich die Symptome von Jugendlichen mit und ohne Posttraumatischer Belastungsstörung (PTBS) unterscheiden. Ein weiteres Ziel der Arbeit ist die vergleichende Analyse der Selbsteinschätzung der Jugendlichen und der Bewertung der Eltern.

Methodik Insgesamt wurden $N=41$ Patienten im Alter von 11-18 Jahren sowie bei jedem Jugendlichen ein Elternteil befragt. Alle Patienten wurden aufgrund akuter Belastung oder nach traumatisierenden Gewalterfahrungen der Forenischen Ambulanz der Universitätsklinik für Kinder- und Jugendpsychiatrie zugewiesen.

Ergebnisse Bezüglich der beiden zusammengefassten Skalen „internalisierende“ und „externalisierende“ Auffälligkeiten beschrieben sich Jugendliche mit PTBS signifikant häufiger auffällig als Jugendliche ohne PTBS. Bei externalisierenden Auffälligkeiten zeigte sich systematisch eine höhere Einschätzung der Verhaltensprobleme der Jugendlichen im Vergleich zu ihren Eltern.

\footnotetext{
S. Völkl-Kernstock $(\bowtie) \cdot$ K. Skala $\cdot$ S. Klomfar ·

O. D. Kothgassner

Universitätsklinik für Kinder- und Jugendpsychiatrie,

Medizinische Universität Wien, Währinger Gürtel

18-20, 1090 Wien, Österreich

sabine.voelkl-kernstock@meduniwien.ac.at

O. D. Kothgassner

Abteilung Klinische Psychologie der Ärztlichen Direktion,

Medizinischer Universitätscampus, Allgemeines

Krankenhaus der Stadt Wien, Wien, Österreich
}

Schlussfolgerungen Die vorliegenden Ergebnisse zeigen eine Divergenz der Eltern-Kind-Einschätzung zu Verhaltensauffälligkeiten auf, die seitens der elterlichen Bezugspersonen nicht in einem klinisch relevanten Ausmaß wahrgenommen werden. Demzufolge ist in der klinischen Praxis bereits am Beginn der Diagnostik bei traumatisierten Kindern und Jugendlichen darauf zu achten, die Sichtweise der Eltern miteinzubeziehen und möglichem elterlichen Missverstehen entgegen $\mathrm{zu}$ wirken.

Schlüsselwörter Trauma - Posttraumatische Belastungsstörung · Jugendliche · CBCL · YSR

\section{The diagnostic relevance of $\mathrm{CBCL}$ and YSR in diagnosing PTSD in adolescence}

\section{Summary}

Background The present study sought to examine the symptomatology of children and adolescents with a history of trauma experience of using the German versions of Child Behavior Checklist (CBCL) and Youth Self Report (YSR). We pursue the question to what extent symptoms differ among adolescents with and without posttraumatic stress disorder (PTSD). A further objective is comparing self-assessment of the children and adolescents with the rating of their parents or caregivers.

Methods Overall $N=41$ patients, aged 11-18 years, and one caregiver of each patient were surveyed. All patients were referred to the forensic ambulance of the University Clinic of Child and Adolescent Psychiatry in consequence of acute burden or violent trauma. Results Concerning the two global Scales ("Internalizing" and "Externalizing" symptoms), adolescents with PTSD reported significantly more problems than adolescents without PTSD diagnosis. Further, results on Externalizing Scale showed a systematic higher prob- 
lem estimation of children and adolescents when compared to their parents.

Conclusions The present findings reveal a divergence between child and parent ratings on clinically relevant behavioral problems. Symptoms experienced by the children and youth are often not being recognized by parents. Therefore, it is essential to include the perceptions of the parents at the beginning of diagnostic assessment and counteract possible parental misunderstanding.

Keywords Trauma - Post-Traumatic Stress Disorders . Adolescents · CBCL · YSR

\section{Einleitung}

Traumatische Erfahrungen im Kindes- und Jugendalter können unterschiedliche schwerwiegende psychische Folgen mit sich bringen. Sexueller Missbrauch erhöht das Risiko für spätere mentale Erkrankungen um das 2,4-fache, bei physischem Missbrauch steigt das Risiko um das 1,5-fache an [22]. Psychische Erkrankungen als wahrscheinliche Folge von Traumata in Kindheit und Adoleszenz beinhalten sowohl internalisierende Probleme wie Depression und Anpassungsstörungen [31, 47, 52] als auch externalisierende Auffälligkeiten wie Probleme mit Peers, aggressives und dissoziales Verhalten [7]. Zudem zeigen misshandelte Kinder und Jugendliche oft Beeinträchtigungen der Exekutivfunktionen (beispielsweise Aufmerksamkeit oder Verarbeitungsgeschwindigkeit) sowie der motorischen und sprachlichen Fähigkeiten $[15,19,45]$ fanden Zusammenhänge zwischen verschiedenen traumatischen Erfahrungen und höheren klinischen Werten bezüglich Depression, Wut, posttraumatischem Stress und Dissoziation während Resilienz-Faktoren verhältnismässig gering ausgeprägt waren. Laut einer erst kürzlich veröffentlichten Untersuchung von Völkl-Kernstock et al. [55] erhielten Kinder, die Erfahrungen häuslicher oder schulischer Gewalt machen mussten, am häufigsten die Diagnosen in den Kategorien Neurotische, Belastungs- und somatoforme Störungen (F4) und Verhaltens- und emotionale Störungen mit Beginn in der Kindheit und Jugend (F9).

Zudem besteht zunehmend Evidenz dafür, dass Kindesmisshandlung und -vernachlässigung einen bedeutenden Risiko-Faktor für die Entwicklung einer PTBS sowie von Dissoziationssymptomen darstellt [15, 28, 52]. Das Auftreten einer Posttraumatischen Belastungsstörung (PTBS) ist nach physischen, und hierbei vor allem nach sexuellen Angriffen mit einer Prävalenzrate von $55 \%$ besonders hoch [31]. Gerade im Kindes- und frühen Jugendalter (unter 14 Jahren) werden traumatische Erfahrungen häufig mit einer PTBS assoziiert [41]. PTBS äußert sich durch vier Hauptsymptom-Cluster: Intrusion, Vermeidung, Negative Veränderungen von Kognition und Stimmung sowie Hyperarousal [4].
Um den gravierenden Auswirkungen von traumatischen Erlebnissen entgegen zu wirken, sind frühzeitige Diagnostik und daran orientierte Interventionsmaßnahmen notwendig. Aufgrund der hohen Komorbiditätsraten von PTBS mit anderen psychischen Erkrankungen [41], empfiehlt sich neben spezifischen diagnostischen Verfahren der zusätzliche Einsatz von Fragebögen wie CBCL und YSR, die ein breites Spektrum an psychischen Auffälligkeiten ressourceneffizient erfassen. Der Elternfragebogen über das Verhalten von Kindern und Jugendlichen (CBCL) und der Fragebogen für Jugendliche (YSR) des Achenbach System of Empirically Based Assessment [3] sind weit verbreitete, kosten- und zeiteffiziente Messinstrumente für eine Reihe von psychischen Auffälligkeiten im Kindes- und Jugendalter. Auch im deutschsprachigen Raum werden sie standardmäßig eingesetzt [2, 8, 9]. Die diskriminante Validität der deutschsprachigen Version des CBCL ist laut Schmeck et al. [51] mit der englischsprachigen Version vergleichbar und weist eine gute Sensitivität $(83,3 \%)$ sowie Spezifität $(83,9 \%)$ auf. Aufgrund der hohen Sensitivität ermöglicht die Diagnostik mittels CBCL ein frühzeitiges Erkennen verschiedener psychischer Probleme. Der „Total Problem Score“ eignet sich zudem ausgezeichnet als Screening für psychische Erkrankungen des Kindes und Jugendalters [51]. Die Vorgabe der CBCL erwies sich bei der Identifikation einiger psychischer Erkrankungen, wie beispielsweise Autismus-Spektrum-Störung [39], ADHS [21], und Zwangsstörungen [49] als nützlich. Obwohl die CBCL-Skalen nicht zur Erfassung von Dissoziation und PTBS entwickelt worden sind, erscheint die Vorgabe der CBCL und des YSR auch bei PTBS im Sinne eines Breitbandscreenings als sinnvoll. So zeigte eine Studie von Gavranidou et al. [25], dass minderjährige Flüchtlinge mit einer PTBS sich auf den Skalen „ängstlich-depressiv“, „schizoid-zwanghaft“, „aggressives Verhalten“, „Internale Auffälligkeiten“ und „Gesamtbelastung“ des YSR signifikant häufiger auffällig beschreiben als Flüchtlinge ohne PTBS. Goldin et al. [26] fanden umfassende Ähnlichkeiten zwischen klinischen Urteilen, welche auf semistrukturierten Interviews mit der Familie basierten und den Urteilen der Bezugsperson (CBCL) und der Grundschulkinder selbst (YSR).

Die Berücksichtigung von verschiedenen Informationsquellen ist in der Diagnostik von psychischen Auffälligkeiten im Kindes- und Jugendalter eine gängige und empfohlene Methode [30]. Da CBCL und YSR vergleichbare Items beinhalten, werden sie häufig im Sinne einer Kreuzvalidierung eingesetzt. Eine Reihe von Untersuchungen beschäftigten sich mit der Übereinstimmung zwischen Elternratings (CBCL) und solchen der Kinder und Jugendlichen selbst (YSR), die vor allem beim Vorhandensein von Auffälligkeiten eher als moderat bis gering $\mathrm{zu}$ bezeichnen ist $[11,27,35,36,44,48,50]$. Schon Plück et al. [44] fanden mittelstarke Zusammenhänge zwischen den Einschätzungen der Jugendlichen und denen der 
Abb. 1 IES-R Werte über die drei Skalen zwischen Patienten mit und ohne PTBS; alle Skalen unterscheiden sich zwischen den Gruppen statistisch signifikant hinsichtlich der dimensionalen Symptomausprägung $(\alpha<$ $0,05)$

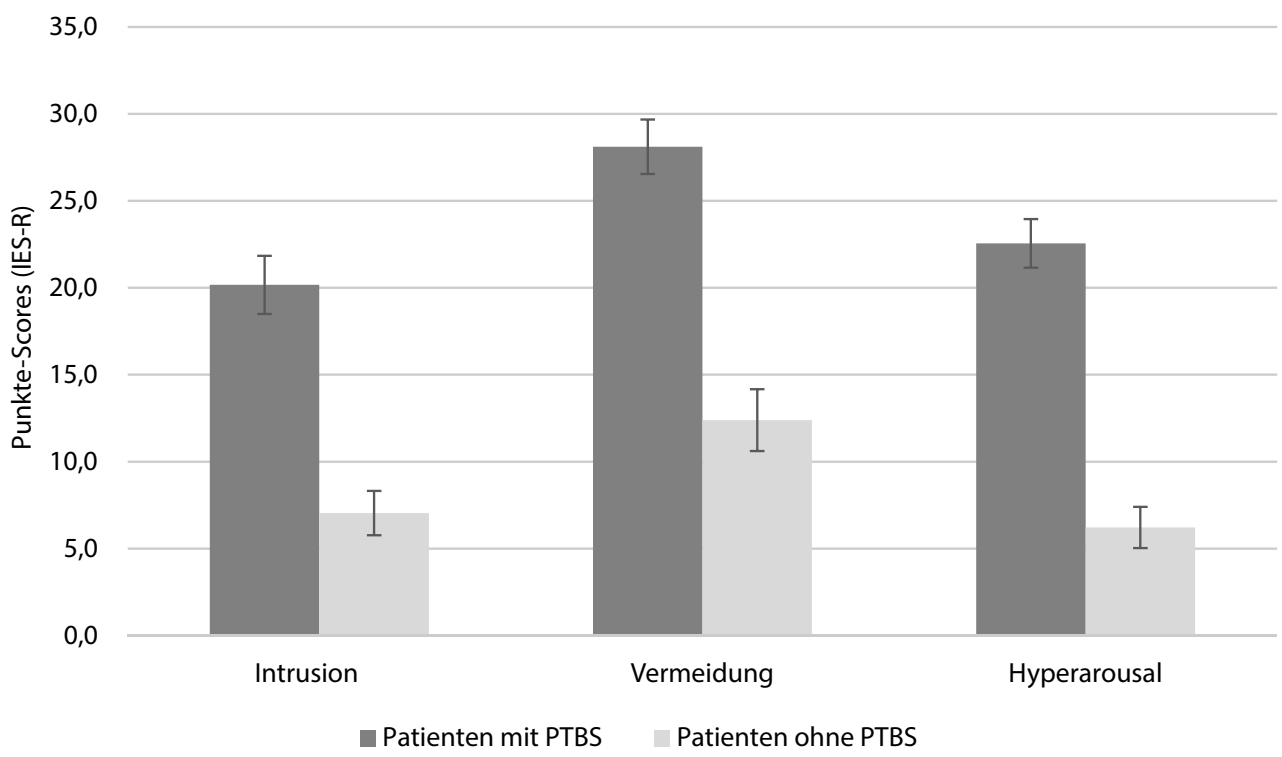

Eltern, bei stark erhöhter Auffälligkeit war eine Übereinstimmung sogar nur in $30 \%$ der Fälle gegeben. Eine in Deutschland durchgeführte Studie fand ebenfalls mittelstarke Eltern-Kind-Übereinstimmungen, besonders niedrig war der Zusammenhang bezüglich der Skala zu kognitiven Schwierigkeiten [11]. Jugendliche aus nicht-klinischen Stichproben schätzen sich in den meisten Untersuchungen auffälliger ein als ihre Eltern dies tun, während sich das Bild bei klinischen Stichproben genau umdreht [34, 36, 43, 44, 48, 59]. Großteils fällt die Übereinstimmung zwischen Selbst- und Fremdurteilen bei externalisierenden Items höher aus als bei internalisierenden [36, 48, 50]. Eine wahrscheinliche Erklärung hierfür liegt darin, dass externalisierende Symptome im Gegensatz $\mathrm{zu}$ internalisierenden leichter beobachtbar sind und daher von Eltern und Lehrern eher bemerkt sowie angegeben werden. Um Ursachen der Beurteilungsdiskrepanzen zu verstehen, wurden einige Studien zu assoziierten Faktoren durchgeführt. Diese fokussierten insbesondere auf Geschlecht und Alter der Kinder bzw. Jugendlichen. Während das Geschlecht für die Eltern-Kind Diskrepanzen irrelevant erscheint, ist die Datenlage zum Einfluss des Alters der Kinder bzw. Jugendlichen inkonsistent [18]. Weitere untersuchte assoziierte Faktoren betreffen unter anderem Depression, Ängste oder Stress der Bezugsperson [34, 59] sowie kontrollierendes Erziehungsverhalten [36]. Trotz der hohen Beurteilungsdiskrepanzen zwischen Kindern bzw. Jugendlichen und deren Eltern ist es laut Achenbach [1] sinnvoll, verschiedene Informanten in den diagnostischen Prozess im Kinder- und Jugendalter miteinzubeziehen, da so unterschiedliche Aspekte des Problems beachtet werden können.

Trotz der weltweiten Verbreitung der CBCL in Forschung sowie Praxis gibt es im deutschsprachigen Raum vergleichsweise wenige Untersuchungen zur Nützlichkeit und Verwendung der CBCL bei PTBS. Sowohl für Forschungszwecke als auch um wirksame, empirisch unterstützte Behandlungen zu entwickeln, ist die ressourceneffiziente Erfassung von PTBS und assoziierten Symptomen bei Kindern und Jugendlichen wichtig. Dadurch stellt sich die Frage, bezüglich welcher Symptomskalen der CBCL bzw. YSR sich Kinder und Jugendliche mit und ohne PTBS unterscheiden, welche in der vorliegenden Studie untersucht werden soll. Es existiert bereits eine Vielzahl an Studien zur Eltern-Kind Übereinstimmung mittels CBCL und YSR, jedoch wurden diese bisher noch nicht unter dem Gesichtspunkt von Traumatisierung untersucht. Ein weiteres Ziel der vorliegenden Arbeit ist daher die vergleichende Analyse der Selbstbeurteilungen von Kindern und Jugendlichen, die eine oder mehrere traumatische Erfahrungen gemacht haben und der Einschätzung deren Eltern bezüglich emotionalen sowie Verhaltensauffälligkeiten anhand der standardisierter Fragebögen CBCL und YSR. Dabei soll der Frage nachgegangen werden, ob und in welchem Ausmaß sich die Übereinstimmungsraten der einzelnen Problemskalen bei Jugendlichen mit PTBS im Vergleich zu Jugendlichen ohne PTBS unterscheiden.

\section{Methode}

Die vorliegende Studie wurde an der Forensischen Ambulanz der Universitätsklinik für Kinder- und Jugendpsychiatrie an der Medizinischen Universität Wien durchgeführt. Die Eltern aller Patienten unterzeichneten vor der Teilnahme an der Untersuchung eine Einwilligungserklärung nach einer ausführlichen Patienteninformation. Ebenso wurden alle befragten Patienten über die Studie vollständig informiert und stimmten einer Teilnahme zu. Die Studie wurde von der zuständigen Ethikkommission begutachtet und mit einem positiven Votum versehen (EK-Nummer: 476/2004). 
Tab. 1 Alter und Diagnosen der Patienten mit und ohne PTBS

\begin{tabular}{l|l|l|l|}
\hline \multirow{2}{*}{ Keine PTBS } & Alter & Diagnosen in \% & \\
& 13,4 & Depressionen & 30,4 \\
\hline & $(1854)$ & Angststörungen & 34,8 \\
\hline PTBS & - & ADHS/SSV & 34,8 \\
\hline & 14,7 & Depressionen & 0 \\
\hline & $(1994)$ & Angststörungen & 83,3 \\
\hline & - & ADHS/SSV & 16,7
\end{tabular}

ADHS Aufmerksamkeitsdefizit-Hyperaktivitätsstörung, SSV Störung des Sozialverhaltens

Tab. 2 CBCL/YSR Skalen geteilt nach internalisierenden und externalisierenden Probleme $(N=41)$

\begin{tabular}{|c|c|c|c|}
\hline & & \multicolumn{2}{|l|}{ Diagnose } \\
\hline & & $\begin{array}{l}\text { Keine PTBS } \\
(n=23)\end{array}$ & $\begin{array}{l}\text { PTBS } \\
(n=18)\end{array}$ \\
\hline \multicolumn{4}{|l|}{ Internalisierende Probleme } \\
\hline \multirow[t]{2}{*}{ Sozialer Rückzug } & Jugendliche & $3,74(3360)$ & $6,22(3422)$ \\
\hline & Eltern & $7,09(4680)$ & $6,44(3650)$ \\
\hline \multirow[t]{2}{*}{ Körperliche Beschwerden } & Jugendliche & 3,17 (2167) & $6,06(4151)$ \\
\hline & Eltern & $4,35(3120)$ & $2,39(2524)$ \\
\hline \multirow[t]{2}{*}{ Angst/Depression } & Jugendliche & $5,74(4298)$ & $15,17(6271)$ \\
\hline & Eltern & $9,39(5922)$ & $11,00(5871)$ \\
\hline \multirow[t]{2}{*}{ Soziale Probleme } & Jugendliche & $2,65(2656)$ & $4,39(3517)$ \\
\hline & Eltern & 3,87 (2989) & $2,67(3515)$ \\
\hline \multirow{2}{*}{$\begin{array}{l}\text { Schizoide, repetitive und } \\
\text { Schlafprobleme }\end{array}$} & Jugendliche & $1,96(1870)$ & $5,06(2510)$ \\
\hline & Eltern & $2,87(2380)$ & 2,28 (1934) \\
\hline \multirow[t]{2}{*}{ Aufmerksamkeitsstörung } & Jugendliche & $5,52(2556)$ & $9,33(3290)$ \\
\hline & Eltern & $8,65(4119)$ & $9,09(3614)$ \\
\hline \multicolumn{4}{|l|}{ Externalisierende Probleme } \\
\hline \multirow[t]{2}{*}{ Delinquentes Verhalten } & Jugendliche & $4,57(2555)$ & $8,44(3914)$ \\
\hline & Eltern & $4,43(4176)$ & $6,67(3956)$ \\
\hline \multirow[t]{2}{*}{ Aggression } & Jugendliche & $7,65(4216)$ & $16,11(9016)$ \\
\hline & Eltern & $11,57(7537)$ & 13,89 (7992) \\
\hline
\end{tabular}

Patienten. Insgesamt wurden 41 Patienten (26 weiblich, 15 männlich) im Alter von 11,0 bis 17,11 ( $\mathrm{M}=$ 14,00 ; SD $=1,988$ ) sowie jeweils ein Elternteil pro Patient (38 Mütter; 3 Väter) befragt. Alle Patienten wurden an die forensische Ambulanz wegen akuter Belastungen oder nach traumatisierenden Gewalterfahrungen zugewiesen. Während bei 18 (13 weiblich, 5 männlich) Patienten im Anschluss an die psychologische Diagnostik ein Posttraumatische Belastungsstörung (PTBS, F43.1) diagnostiziert wurde, war dieses bei 23 (13 weiblich, 10 männlich) nicht der Fall. Die Patienten wurden im Zeitraum zwischen 1 Monat und 6 Monaten nach dem traumatisierenden Ereignis diagnostiziert, neben einer ausgiebigen Exploration wurden Fragebogenverfahren unter zusätzlicher zur Hilfenahme strukturierter klinischer Interviews (SKID-I; [57]) durchgeführt, um die Diagnose abzusichern. Während Abb. 1 die dimensionale Symptomausprägung der beiden sich daraus ergebenden Gruppen (Patien- ten mit PTBS und ohne PTBS) nach dem IES-R [37] zeigt, illustriert Tab. 1 die Häufigkeit von Komorbiditäten oder anderen Diagnosen nach ICD-10 nach den beiden Untersuchungsgruppen aufgeschlüsselt und das durchschnittliche Alter der Patienten innerhalb der Gruppen. Patienten, welche keine ausreichenden Kenntnisse der deutschen Sprache aufweisen konnten, sowie Patienten, die an einer anderen körperlichen oder einer diagnostizierten psychiatrischen Erkrankung vor dem Ereignis litten, wurden nicht in die Studie eingeschlossen.

Selbst- und Fremdratings. Im Rahmen einer standardisierten psychologischen Untersuchung wurden Jugendlichen und Eltern Selbst- und Fremdbeurteilungsbögen ausgegeben.

\section{Verwendete Instrumente}

CBCL: Die Child Behavior Checklist (CBCL) stellt eine Fremdbeurteilung von Kindern und Jugendlichen im Alter von 6-18 Jahren durch die Eltern zur Erfassung von Verhaltensauffälligkeiten, sowie sozialen und emotionalen Problemen, aber auch körperlichen Beschwerden dar. Eine passende Form zur Selbstbeurteilung für Jugendliche von 11-18 ist direkt von der CBCL abgeleitet. Beide Beurteilungsverfahren beinhalten die internalisierenden Skalen a) Sozialer Rückzug, b) körperliche Beschwerden, c) Angst/Depression, d) soziale Probleme, e) schizoide, repetitive und Schlafprobleme, sowie d) Aufmerksamkeitsschwierigkeiten; darüber hinaus inkludieren die Verfahren externalisierende Verhaltensweisen wie e) delinquentes, oppositionelles Verhalten und f) aggressives Verhalten. Eine zusätzliche Skala im YSR erfragt selbstverletzende und selbstschädigende Verhaltensweisen (Autoaggression). Um das multifaktorielle Geschehen bei PTBS auf den Symptomskalen der CBCL/YSR abbilden zu können wurden in der Folge internalisierende und externalisierende Symptome bei Jugendlichen und deren Eltern mit und ohne einer PTBS-Diagnose direkt verglichen, was durch die beiden Hauptskalen des CBCL/YSR ermöglicht wird (internalisierende vs. externalisierende Verhaltensweisen).

\section{Statistik}

Die statistische Analyse für die Berechnung der Unterschiede in der Symptombeschreibung der Jugendlichen mit und ohne PTBS, sowie deren Eltern wurde mittels einer Varianzanalyse für abhängige Stichproben mit Diagnose (PTBS vs. keine PTBS) als Zwischensubjektfaktor durchgeführt. Alle statistischen Überprüfungen wurden mittels SPSS 20 (IBM) und auf Basis eines Alphafehlers von $5 \%$ kalkuliert. 
Tab. 3 Mittelwerte und Standardabweichung der Hauptskalen und der Zusatzskala Autoaggressivität

\begin{tabular}{|l|l|l|}
\hline & $\begin{array}{l}\text { Diagnose } \\
\text { Keine PTBS }\end{array}$ & PTBS \\
\hline $\begin{array}{l}\text { Internalisierende Probleme } \\
\text { Jugendliche }\end{array}$ & $12,08(2007)$ & $26,33(2269)$ \\
\hline Eltern & $19,60(2255)$ & $18,67(2549)$ \\
\hline Externalisierende Probleme & & \\
\hline Jugendliche & $61,00(4598)$ & $104,11(5194)$ \\
\hline Eltern & $59,45(5246)$ & $61,39(5800)$ \\
\hline Autoaggressivität & & \\
\hline Jugendliche & $2,50(2081)$ & $7,78(4917)$ \\
\hline
\end{tabular}

\section{Ergebnisse}

Tab. 2 zeigt die Mittelwerte und Standardabweichungen der Selbst- und Fremdbeurteilungsbögen bei internalisierenden und externalisierenden Symptomen.

In der Skala Sozialer Rückzug konnte ein signifikanter Unterschied zwischen den Symptombeschreibungen der Jugendlichen und der Eltern festgestellt werden $\left(F(1,39)=5442 ; p=0,025\right.$; par. $\left.\eta^{2}=0,122\right)$. Keinen Effekt hatte hingegen die Diagnose der Jugendlichen auf die Einschätzung des sozialen Rückzugs $(F(1,39)=$ 0,$960 ; p=0,333$; par. $\eta^{2}=0,024$ ). Diese Ergebnisse sind jedoch aufgrund des signifikanten Wechselwirkungseffektes $\left(F(1,39)=4,181 ; p=0,048\right.$; par. $\left.\eta^{2}=0,097\right)$ dahingehend $\mathrm{zu}$ sehen, dass besonders Jugendliche ohne PTBS-Diagnose niedrigere Werte im sozialen Rückzug berichten als ihre Eltern. Hinsichtlich der Körperlichen Beschwerden sind nur bei einer Erhöhung des Alphaniveaus auf $10 \%$ signifikante Unterschiede zwischen Jugendlichen und Eltern $(F(1,39)=4003$; $p=0,052$; par. $\left.\eta^{2}=0,093\right)$ festzustellen, sowie kein signifikanter Unterschied hinsichtlich der Diagnose $\left(F(1,39)=0,395 ; p=0,533 ; \eta^{2}=0,010\right)$ zu beobachten. In der Skala Angst und Depression gibt es keine signifikanten Unterschiede hinsichtlich den Bewertungen der Jugendlichen und deren Eltern $(F(1,39)=0,890 ; p=$ 0,767 ; par. $\left.\eta^{2}=0,002\right)$. Ein signifikanter Unterschied ist jedoch zwischen den Diagnosegruppen festzustellen $\left(F(1,39)=11.521 ; p=0,002 ; \eta^{2}=0,002\right)$, wobei Jugendliche mit PTBS und deren Eltern die Symptome von Angst und Depression signifikant negativer beschreiben, als Jugendliche ohne PTBS und deren Eltern. Eine signifikante Wechselwirkung zeigt an, dass Eltern von Jugendlichen mit PTBS höhere Werte angeben, als Eltern von Jugendlichen ohne PTBS $(F(1,39)=$ 20.652; $p<0,001$; par. $\left.\eta^{2}=0,346\right)$. Keine signifikanten Unterschiede zwischen Jugendlichen und deren Eltern ergeben sich auch hinsichtlich der Bewertung sozialer Probleme $\left(F(1,39)=0,167 ; p=0,685\right.$; par. $\eta^{2}=$ 0,004). Auch hinsichtlich der Jugendlichen mit und ohne PTBS konnten keine signifikanten Unterschiede festgestellt werden $(F(1,39)=0,958 ; p=0,334$; par. $\left.\eta^{2}=0,024\right)$. Die Angaben von Jugendlichen und ihren Eltern unterscheiden sich in der Skala schizoide, re- petitive und Schlafprobleme nur bei einer Erhöhung des Alphaniveaus auf $10 \%$ signifikant $(F(1,39)=3467$; $p=0,070$; par. $\left.\eta^{2}=0,082\right)$, jedoch gibt es signifikante Unterschiede zwischen den Bewertungen hinsichtlich der Diagnose in der Beschreibung von Jugendlichen und deren Eltern $(F(1,39)=7087 ; p=0,011$; par. $\left.\eta^{2}=0,154\right)$. Ein signifikanter Wechselwirkungseffekt $\left(F(1,39)=13.583\right.$; $p=0,001$; par. $\left.\eta^{2}=0,258\right)$ deutet darauf hin, dass besonders Jugendliche mit PTBS mehr schizioide, repetitive und Schlafprobleme berichten als andere Jugendliche ohne PTBS. Darüber hinaus sind die Beschreibungen einer Aufmerksamkeitsstörung bei Jugendlichen und deren Eltern signifikant unterschiedlich $(F(1,39)=5096 ; p=0,030$; par. $\eta^{2}=0,116$ ), wobei Jugendliche niedrigere Werte selbst berichten. Dieses Ergebnis ist jedoch besonders auch in Bezug darauf zu interpretieren, dass signifikante Unterschiede zwischen den Berichten über oder von einem Patienten mit oder ohne PTBS-Diagnose $\mathrm{zu}$ beobachten sind $(F(1,39)=5489 ; p=0,024$; par. $\left.\eta^{2}=0,123\right)$. Ein signifikanter Wechselwirkungseffekt $\left(F(1,39)=7815\right.$; $p=0,008$; par. $\left.\eta^{2}=0,167\right)$ deutet darauf hin, dass besonders Jugendliche ohne PTBS sich wesentlich geringer einstufen, wie ihre Eltern oder Jugendliche und ihre Eltern mit PTBS.

Neben den gerade beschriebenen internalen Symptomen, sind auch externalisierende Symptome bei PTBS vielfach vorherrschend. Hinsichtlich delinquentem Verhalten ergeben sich keine signifikanten Unterschiede zwischen den Berichten der Jugendlichen und deren Eltern $\left(F(1,39)=1712 ; p=0,198\right.$; par. $\eta^{2}=$ 0,042 ), jedoch gibt es einen signifikanten Unterschied zwischen den Berichten von Jugendlichen mit PTBS und den Jugendlichen ohne PTBS $(F(1,39)=11.582$; $p=0,002$; par. $\left.\eta^{2}=0,229\right)$, wobei Jugendliche mit PTBS deutlich höheres delinquentes Verhalten zeichnen. Bezüglich der Aggressionsneigung zeigen sich erneut keine Unterschiede zwischen den Selbst- und Fremdbeurteilungen bei Jugendlichen und deren Eltern $\left(F(1,39)=0,523 ; p=0,474\right.$; par. $\left.\eta^{2}=0,013\right)$, jedoch auch hier zeigen Jugendliche mit PTBS erhöhte Werte in Selbst- und Fremdbeurteilung $(F(1,39)=7551$; $p=0,009$; par. $\left.\eta^{2}=0,162\right)$. Der Wechselwirkungseffekt $\left(F(1,39)=6885 ; p=0,012\right.$; par. $\left.\eta^{2}=0,150\right)$ zeigt, dass besonders die Selbstberichte große Differenzen aufzeigen und so vor allem Jugendliche mit PTBS deutlich höhere Aggressionswerte berichten.

In der ersten Hauptskala des CBCL/YSR zeigt sich, dass Jugendlichen und Eltern bei internalisierenden Verhaltensweisen keine signifikanten Unterschiede berichten $\left(F(1,39)=7551 ; p=0,967\right.$; par. $\left.\eta^{2}=0,000\right)$, jedoch signifikante Unterschiede zwischen Jugendlichen mit und ohne PTBS auftreten $(F(1,39)=6047$; $p=0,018$; par. $\eta^{2}=0,134$ ). Die zweite Hauptskala zu den externalisierenden Verhaltensweisen wiederum ergibt signifikante Unterschiede zwischen der Selbstund Fremdeinschätzung $(F(1,38)=296.297 ; p<0,001$; par. $\eta^{2}=0,886$ ), sowie auch signifikante Unterschiede zwischen den Beschreibungen der Jugendlichen mit 
und ohne PTBS $\left(F(1,39)=33.212 ; p<0,001\right.$; par. $\eta^{2}=$ 0,466). Der Wechselwirkungseffekt zeigt an, dass besonders bei Jugendlichen mit PTBS unterschiedliche Selbst- und Fremdbeschreibungen hinsichtlich ihrem delinquenten und aggressiven Verhalten $\mathrm{zu}$ finden sind $\left(F(1,38)=27 \cdot 047 ; p<0,001\right.$; par. $\left.\eta^{2}=0,421\right)$. Die zusätzliche Skala für Jugendliche Autoaggression zeigt hier einen signifikanten Unterschied zwischen Jugendlichen mit und ohne PTBS $(F(1,40)=21.464$; $p<0,001)$. Tab. 3 zeigt alle Mittelwerte und Standardabweichungen der Hauptskalen und der zusätzlichen Skala.

\section{Diskussion}

Die Lebenszeitprävalenz der posttraumatischen Belastungsstörung liegt bei $7-8 \%$, bei den unter 18 jährigen beträgt diese $1,6 \%$ [20, 23, 32, 40, 53]. Die Hälfte der Betroffenen erfährt eine Reduktion der Symptomatik der Belastungsstörung innerhalb von 12 Monaten, $15 \%$ der Betroffenen hingegen zeigen trotz Therapie eine Chronifizierung der posttraumatischen Störung. Klinische Befragungen zeigen eine Prävalenz von etwa 3,5\% der Gesamtbevölkerung irgendeine Form von Belastungsstörung betreffend [17, 42]. Weibliche Personen haben ein etwa doppelt so hohes Risiko, eine Belastungsstörung zu entwickeln als männliche das heißt während bis $\mathrm{zu} 20 \%$ der traumaexponierten Frauen Symptome im Sinne einer PTBS entwickeln, ist dies nur bei etwa $8 \%$ der traumaexponierten Männer der Fall [33]. Bezugnehmend auf das Erleben physischer und sexueller Gewalt entwickeln mehr als 1/3 aller Opfer eine posttraumatische Belastungsreaktion [29]. Lang andauernde, chronifizierte Misshandlungen und Missbrauch in der Familie führen häufig zur Entwicklung psychischer Störungen wie Depression, Suchtverhalten und Persönlichkeitsstörungen [14].

Diese Daten implizieren, dass man trotz umfassenden klinischen Wissens über PTBS und mögliche Folgen bei Gewalterfahrungen von Kindern und Jugendlichen noch sensibler und differenzierter im Bereich der klinischen Diagnostik vorgehen sollten. Als unerlässlich gilt bei Kinder und Jugendlichen, deren elterliche Betreuungspersonen und deren Sichtweisen und Wahrnehmungen in den Prozess der Diagnostik einzubeziehen, da sieeine maßgebliche Ressource oder aber auch einen Verstärker des Stresserlebens für ihre Kinder darstellen können [6, 13].

Erwähnenswert für die Diskussion der vorliegenden Ergebnisse ist, dass Traumatisierungen nicht nur als beobachtbares Ereignis zu erachten sind, sondern vielmehr aus der Perspektive der subjektiven Realität des Opfers verstanden werden müssen [58], die bei dem Erleben eines Traumas aus der völligen Hilflosigkeit, Ohnmacht und Gefühlen des Ausgeliefertseins besteht. Manche Patienten sind trotz massiver Traumatisierung in der Kindheit und Jugend erstaunlich wenig geschädigt, andere wurden „objektiv“ betrach- tet weit weniger intensiv traumatisiert, zeigen aber eine starke Folgesymptomatik. Inwieweit ein Trauma die weitere Entwicklung beeinflusst, ist neben klassischen Resilienzfaktoren wie Geschlecht, Temperament und Intelligenz auch von Alter, Entwicklungsstand, Ausmaß der Traumatisierung und dem sozialen Umfeld abhängig [12, 16].

Im Sinne einer orientierenden präzisierenden Diagnostik sind in einem ersten Schritt etwaige psychoreaktive Auffälligkeiten, welche in Zusammenhang mit der Erfahrung von Gewalt stehen können, abzuklären. Screening-Instrumente zur ökonomischen fremd- sowie eigenanamnestischen Erfassung sind „Child Behavior Checklist“ (CBCL/11/2-5, CBCL/4-18; [2, 8]) oder der „Fragebogen für Jugendliche“ (YSR; [9]).

Die Tatsache, dass von uns untersuchte Jugendliche mit einer PTBS sich von Jugendlichen ohne PTBS hinsichtlich ihrer depressiven und ängstlichen Symptomatik, sowie bezüglich schizoidem, zwanghaften Verhalten und Schlafproblemen unterscheiden ist konsistent mit den ICD-10 Kriterien für eine PTBS. Hierbei ist zu erwähnen, dass oftmals intrusive Inhalte im Screening als schizoid gedeutet werden können, aktuelle Studien aber auch die positiven, Zusammenhänge zwischen psychotischen Symptomen, PTBS und früher Traumatisierung, sowie die Ontogenese repetitiver Symptomatik durch frühe Trauma bereits abgehandelt haben (u. a. [38, 46]).

Ebenfalls geben Jugendliche mit PTBS höhere Probleme in ihrer Aufmerksamkeit an. Dies ist jedoch insbesondere unter dem differentialdiagnostischen Aspekt zu sehen. Dabei ist wesentlich zu differenzieren, welche Anteile der Aufmerksamkeitsproblematik einer eventuellen komorbiden Aufmerksamkeitsdefizit-Hyperaktivitätsstörung (ADHS) anzulasten sind und welche sich durch die akute Belastungssituation bzw. die posttraumatischen weiteren Entwicklungen erklären lassen. Interessant ist jedenfalls, dass besonders Eltern der Jugendlichen ohne PTBS die Symptomatik stärkere Aufmerksamkeitsstörungen berichten, als die betroffenen Jugendlichen selbst. Dies könnte damit in Zusammenhang stehen, dass in dieser Gruppe viele Primärdiagnosen $\mathrm{ADHS}$, Depression und Angststörung vergeben wurden und diese Differenzen zwischen Selbst- und Fremdbeschreibung bereits beschrieben wurde [34, 36, 43, 44, 48, 59]. Dieselbe Argumentation trifft ebenfalls auf die Skala sozialen Rückzug zu, da besonders Jugendliche ohne PTBS Diagnose als weniger sozial zurückgezogen beschreiben, wie ihre Eltern sie beschreiben würden und auch sich Jugendliche mit PTBS beschreiben. Demzufolge ist aus diesem Ergebnis ableitbar, dass Eltern von Jugendlichen mit Gewalterfahrungen, allerdings ohne diagnostizierter PTBS, die emotionale Situation ihrer Kinder nicht entsprechend dem Erleben ihrer Kinder wahrzunehmen scheinen und lediglich einen sozialen Rückzug ohne behandlungsbedürftiger Relevanz bei diesen bemerken, der möglicherweise auch als (prä-) adoleszentes Verhalten interpretiert werden könnte. 
Jedenfalls scheint es, als wären die Symptomgruppen Aufmerksamkeitsprobleme und sozialer Rückzug bei Jugendlichen mit PTBS konsistent mit der Wahrnehmung der Eltern beschrieben.

Keine Unterscheidungen hinsichtlich Diagnose PTBS vs. keine PTBS, sowie auch nicht im Selbstund Fremdrating ergaben sich in den Skalen Soziale Probleme, sowie körperlicher Beschwerden. Dies muss ebenfalls kritisch in Zusammenhang mit den Primärdiagnosen der Vergleichsstichprobe gesehen werden, da alle Jugendliche darin ausgeprägte Psychopathologien aufwiesen. Besonders im Hinblick auf die sozialen Probleme scheint - wie generell für psychische Störungen - ein funktionierendes soziales Umfeld in Abhängigkeit auch vom Ausmaß der Traumatisierung ein grundlegender Schutzfaktor für PTBS zu sein [16].

Hinsichtlich delinquenten und aggressiven Verhaltensweisen bleibt $\mathrm{zu}$ berichten, dass sich besonders Jugendliche mit PTBS als delinquenter und aggressiver beschreiben. Die Beschreibungen der Jugendlichen decken sich dabei mit den Beschreibungen der externalisierenden Verhaltensweisen der Eltern. Eine mögliche Erklärung dafür ist, dass durch das traumatisierende Ereignis eine Schockstarre ausgelöst wird, welche später mit Scham und Schuld bei den betroffenen Jugendlichen behaftet wird. Diese transferiert sich in aggressives Verhalten [5, 54]. Dieses Ergebnis kann in einer längerfristigen Betrachtung auch in Beziehungen zum psychoanalytisch-bindungstheoretischen Ansatz Fonagy et al. [24] gebracht werden. Dieser geht davon aus, dass ein sicheres Bindungsmuster in der Kindheit mit der Fähigkeit zur Mentalisierung zusammenhängt. Eine Eigenschaft, welche das verstehen der eigenen Person und anderer Personen ermöglicht und vielen delinquenten Jugendlichen und besonders schweren Straftätern fehlt.

In den Gesamtskalen unterschieden sich Jugendliche mit PTBS und Jugendliche ohne PTBS signifikant voneinander - sowohl hinsichtlich internalisierender, als auch externalisierender Verhaltensweisen, was anhand der Einzelskalen bereits ersichtlich war. Eltern von Jugendlichen mit PTBS zeigen hingegen eine geringere Konsistenz in der Beurteilung externalisierender Verhaltensweisen in der Hauptskala, jedoch hinsichtlich der Hauptskala zu den internalisierenden Verhaltensweisen lagen keine signifikanten Unterschiede vor. Der Unterschied zwischen Eltern und Jugendlichen in der Hauptskala könnte zum Einen dadurch erklärt werden, dass Eltern tatsächlich die aggressiven Verhaltensweisen wahrnehmen, diese jedoch aufgrund der sozialen Tabuisierung von delinquenten und aggressiven Verhaltensweisen abschwächen und ihr Kind somit vor einer zusätzlichen Stigmatisierung schützen wollen. Zum anderen wäre es auch möglich, dass den Eltern die emotionale Belastung der Kinder nicht im ganzen Spektrum wahrnehmen können, die betroffenen Jugendlichen hingegen sehr wohl etwa eine erhöhte Aggressionsneigung ver- spüren und diese auch angeben. Ebenso scheint es, dass Jugendliche mit PTBS auch eine erhöhte Neigung zur Autoaggression aufweisen, was in Einklang mit einer Reihe weiterer Studien steht und besonders oft der Vermeidung der emotionalen Taubheit und Dysphorie dient, welche als Begleitsymptomatik der PTBS oft genannt sind $[10,56]$.

Traumatische Erlebnisse müssen im Laufe der Entwicklung immer wieder neu bearbeitet werden und therapeutische Interventionen müssen einem multimodalen Konzept folgen. Dies gelingt nur über stabile und korrigierende Beziehungserfahrungen (siehe auch [24]). Die Unterstützung des sozialen Umfelds ist daher von wesentlicher Bedeutung, nicht zuletzt um unnötige Beziehungsabbrüchen, die möglicherweise erneut destabilisieren, zu vermeiden.

In der Betrachtung der vorliegenden Ergebnisse erscheint es folglich als wichtig, bereits am Beginn von Diagnostik und Therapie, die Wahrnehmungen der Eltern in Bezug auf das kindliche Verhalten $\mathrm{zu}$ fokussieren und darauf $\mathrm{zu}$ achten, dass elterliches Missverstehen von kindlichem Verhalten eine symptombegünstigende Wirkung haben kann. Auch wenn der Schluss, dass eine hohe Korrelation zwischen kindlicher und elterlicher Wahrnehmung der psychischen Situationen der Betroffenen, an sich einen protektiven Faktor darstellt, nicht zulässig ist, kann ein diesbezügliches Entgegenwirken durch ein verstehendes klinisches „Helfersystem“ Kindern und deren Familien, die von Gewalt betroffen sind, möglicherweise eine wichtige Grundlage zur Etablierung resilienten Verhaltens bieten.

Open access funding provided by Medical University of Vienna.

Interessenkonflikt S. Völkl-Kernstock, K. Skala, S. Klomfar und O.D. Kothgassner geben an, dass kein Interessenkonflikt besteht.

Open Access Dieser Artikel wird unter der Creative Commons Namensnennung 4.0 International Lizenz (http:// creativecommons.org/licenses/by/4.0/deed.de) veröffentlicht, welche die Nutzung, Vervielfältigung, Bearbeitung, Verbreitung und Wiedergabe in jeglichem Medium und Format erlaubt, sofern Sie den/die ursprünglichen Autor(en) und die Quelle ordnungsgemäß nennen, einen Link zur Creative Commons Lizenz beifügen und angeben, ob Änderungen vorgenommen wurden.

\section{Literatur}

1. Achenbach TM. Diagnosis, assessment, and comorbidity in psychosocial treatment research. J Abnorm Child Psychol. 1995;23(1):45-65.

2. Achenbach TM. CBCL 1 1/2-5 Child Behavior Checklist 1 1/2-5-Deutsche Fassung. Elternfragebogen für Klein-und Vorschulkinder. Göttingen: Hogrefe; 2000.

3. Achenbach TM, Rescorla LA. Manual for the ASEBA schoolAge forms \& profiles. Burlington: University of Vermont, Research Center for Children, Youth, and Families; 2001. 
4. American Psychiatric Association. Diagnostic and statistical manual of mental disorders (DSM- $5^{\circledR}$ ). Arlington: American Psychiatric Association; 2013.

5. Andrews B, Brewin CR, Rose S, Kirk M. Predicting PTSD symptoms in victims of violent crime: the role of shame, anger, and childhood abuse. J Abnorm Psychol. 2000;109(1):69-73.

6. Angold A, Messer SC, Stangl D, Farmer EM, Costello EJ, Burns BJ. Perceived parental burden and service use for child and adolescent psychiatric disorders. Am J Public Health. 1998;88(1):75-80.

7. Anthonysamy A, Zimmer-Gembeck MJ. Peer status and behaviors of maltreated children and their classmates in the early years of school. Child Abuse Negl. 2007;31(9):971-91.

8. Arbeitsgruppe Deutsche Child Behavior Checklist. Elternfragebogen über das Verhalten von Kindern und Jugendlichen. Deutsche Bearbeitung der Child Behavior Checklist (CBCL/4-18). Köln: Arbeitsgruppe Kinder-, Jugend- und Familiendiagnostik; 1998.

9. Arbeitsgruppe Deutsche Child Behavior Checklist. Fragebogen für Jugendliche. Deutsche Bearbeitung des Youth Self-Report (YSR) der Child Behavior Checklist.Einführung und Anleitung zur Handauswertung. Köln: Arbeitsgruppe Kinder-, Jugend- und Familiendiagnostik; 1998.

10. Asmundson GJG, Stapleton JA, Taylor S. Are avoidance and numbing distinct PTSD symptom clusters? J Trauma Stress. 2004;17:467-75.

11. Barkmann C, Schulte-Markwort M. Emotional and behavioral problems of children and adolescents in Germany. Soc Psychiatry Psychiatr Epidemiol. 2005;40(5):357-66.

12. Bogyi G. Traumatisierung im Kindes-und Jugendalter. Pädiatr Pädol. 2011;46(4):34-7.

13. Borawski EA, Ievers-Landis CE, Lovegreen LD, Trapl ES. Parental monitoring, negotiated unsupervised time, and parental trust: the role of perceived parenting practices in adolescent health risk behaviors. J Adolesc Health. 2003;33(2):60-70.

14. Borger SC, Cox BJ, Asmundson GJG. PTSD and other mental health problems in adults who report histories of severe physical abuse and neglect. In: Corales TA (Hrsg.). Trends in posttraumatic stress disorder research. New York: Nova Science Publishers; 2005. S. 249-61.

15. Collin-Vézina D, Coleman K, Milne L, Sell J, Daigneault I. Trauma experiences, maltreatment-related impairments, and resilience among child welfare youth in residential care. Int J Ment Health Addict. 2011;9(5):577-89.

16. Copeland WE, Keeler G, Angold A, Costello EJ. Traumatic events and posttraumatic stress in childhood. Arch Gen Psychiatry. 2007;64(5):577-84.

17. Corbin TJ, Purtle J, Rich LJ, Rich JA, Adams EJ, Yee G, et al. The prevalence of trauma and childhood adversity in an urban, hospital-based violence intervention program. J Health Care Poor Underserved. 2013;24(3):1021-30

18. De Los Reyes A, Kazdin AE. Informant discrepancies in the assessment of childhood psychopathology: a critical review, theoretical framework, and recommendations for further study. Psychol Bull. 2005;131(4):483-509.

19. DePrince AP, Weinzierl KM, Combs MD. Executive function performance and trauma exposure in a community sample of children. Child Abuse Negl. 2009;33(6):353-61.

20. De Young AC, Kenardy JA, Cobham VE. Trauma in early childhood: a neglected population. Clin Child Fam Psychol Rev. 2011;14(3):231-50.

21. Eiraldi RB, Power TJ, Karustis JL, Goldstein SG. Assessing ADHD and comorbid disorders in children: the Child Behavior Checklist and the Devereux Scales of Mental Disorders. JClin Child Psychol.2000;29(1):3-16.
22. Fergusson DM, Boden JM, Horwood LJ. Exposure to childhood sexual and physical abuse and adjustment in early adulthood. Child Abuse Negl. 2008;32(6):607-19.

23. Fletcher S, Creamer M, Forbes D. Preventing post traumatic stress disorder: are drugs the answer? Aust NZJ Psychiatry. 2010;44(12):1064-71.

24. Fonagy P, Gergely G, Jurist EL, Target M. Affect regulation, mentalization and the development of the self. London: Karnac books; 2004.

25. Gavranidou M, Niemiec B, Magg B, Rosner R. Traumatische Erfahrungen, aktuelle Lebensbedingungen im Exil und psychische Belastung junger Flüchtlinge. Kindh Entwickl. 2008;17(4):224-31.

26. Goldin S, Hägglöf B, Levin L, Persson LÅ. Mental health of Bosnian refugee children: a comparison of clinician appraisal with parent, child and teacher reports. Nord JPsychiatry. 2008;62(3):204-16.

27. Grigorenko EL, Geiser C, Slobodskaya HR, Francis DJ. Cross-informant symptoms from CBCL, TRF, and YSR: trait and method variance in a normative sample of Russian youths. Psychol Assess. 2010;22(4):893-911.

28. Hulette AC, Freyd JJ, Pears KC, Kim HK, Fisher PA, BeckerBlease KA. Dissociation and posttraumatic symptoms in maltreated preschool children. J Child Adolesc Trauma. 2008;1(2):93-108.

29. Jaycox LH, Foa EB. Posttraumatic stress. In: Friedman HS (Hrsg.). Speciality articles from the encyclopedia of mental health. San Diego:Academic Press; 2001.S.337-46.

30. Jensen PS, Rubio-Stipec M, Canino G, Bird HR, Dulcan MK, Schwab-Stone ME, et al. Parent and child contributions to diagnosis of mental disorder: are both informants always necessary? J Am Acad Child Adolesc Psychiatry. 1999;38(12):1569-79.

31. Johnson J, Maxwell A, Galea S. The epidemiology of posttraumatic stress disorder. Psychiatr Ann. 2009;39(6):326. doi:10.3928/00485713-20090514-01.

32. Kessler RC, Angermeyer M, Anthony JC, De Graaf R, Demyttenaere K, Gasquet I, et al. Lifetime prevalence and age-of-onset distributions of mental disorders in the World Health Organization's World Mental Health Survey Initiative. World Psychiatry. 2007;6(3):168-76.

33. Khouzam HR, Ghafoori B, Hierholzer R. Progress in the identification, diagnosis and treatment of posttraumatic stress disorder. In: Corales TA (Hrsg.). Trends in posttraumatic stress disorder research. New York: Nova Science; 2005. S. 1-28.

34. Kolko DJ, Kazdin AE. Emotional/behavioral problems in clinic and nonclinic children: correspondence among child, parentand teacher reports. JChild PsycholPsychiatry. 1993;34(6):991-1006.

35. Lacalle M, Ezpeleta L, Doménech JM. DSM-oriented scales of the child behavior checklist and youth self-report in clinically referred Spanish children. Span J Psychol. 2012;15(01):377-87.

36. Lohaus A, Vierhaus M. Parent-child discrepancies in the assessment of internalizing/externalizing behavior. Z Entwicklungspsychol Pädagog Psychol. 2014;46(1):1-10.

37. Maercker A, Schützwohl M. Erfassung von psychischen Belastungsfolgen: Die Impact of Event Skala-revidierte Version (IES-R). Diagnostica. 1998;44(3):130-41.

38. Mathews CA, Kaur N, Stein MB. Childhood trauma and obsessive-compulsive symptoms. Depress Anxiety. 2008;25(9):742-51.

39. Muratori F, Narzisi A, Tancredi R, Cosenza A, Calugi S, Saviozzi I, et al. The CBCL 1.5-5 and the identification of preschoolers with autism in Italy. Epidemiol Psychiatr Sci. 2011;20(04):329-38. 
40. Pace TW, Heim CM. A short review on the psychoneuroimmunology of posttraumatic stress disorder: from risk factors to medical comorbidities. Brain Behav Immun. 2011;25(1):6-13.

41. Perkonigg A, Kessler RC, Storz S, Wittchen HU. Traumatic events and post-traumatic stress disorder in the community: prevalence, risk factors and comorbidity. Acta Psychiatr Scand. 2000;101(1):46-59.

42. Perrin M, Vandeleur CL, Castelao E, Rothen S, Glaus J, Vollenweider P, et al. Determinants of the development of post-traumatic stress disorder, in the general population. Soc Psychiatry Psychiatr Epidemiol. 2014;49(3):447-57.

43. Phares V, Danforth JS. Adolescents', parents', and teachers' distress over adolescents' behavior.JAbnorm Child Psychol. 1994;22(6):721-32.

44. Plück J, Döpfner M, Berner W, Fegert JM, Huss M, Lenz K, et al. Die Bedeutung unterschiedlicher Informationsquellen bei der Beurteilung psychischer Störungenim Jugendalter-Ein Vergleich von Elternurteil und Selbsteinschätzung der Jugendlichen. Prax Kinderpsychol Kinderpsychiatr. 1997;46(8):566-82.

45. Prasad MR, Kramer LA, Ewing-Cobbs L. Cognitive and neuroimaging findings in physically abused preschoolers. Arch Dis Child. 2005;90(1):82-5.

46. Powers A, Fani N, Cross D, Ressler KJ, Bradley B. Childhood trauma, PTSD, and psychosis: findings from a highly traumatized, minoritysample.ChildAbuse Negl.2016;58:111-8.

47. Putnam FW. Ten-year research update review: child sexual abuse. J Am Acad Child Adolesc Psychiatry. 2003;42(3):269-78.

48. ReyJM, Schrader E, Morris-Yates A. Parent-child agreement on children's behaviours reported by the child behaviour checklist (CBCL). JAdolesc. 1992;15(3):219-30.

49. RössnerV,BeckerA, RothenbergerA.Psychopathologisches Profil bei Tic-und Zwangsstörungen. Kindh Entwickl. 2007;16(2):110-6.

50. Salbach-Andrae H, Lenz K, Lehmkuhl U. Patterns of agreement among parent, teacher and youth ratings in a referred sample. Eur Psychiatry. 2009;24(5):345-51.
51. Schmeck K, Poustka F, Döpfner M, Plück J, Berner W, Lehmkuhl G, et al. Discriminant validity of the child behaviour checklist CBCL-4/18 in German samples. Eur Child Adolesc Psychiatry. 2001;10(4):240-7.

52. Silva RR, Alpert M, Munoz DM, Singh S, Matzner F, Dummit S. Stress and vulnerability to posttraumatic stress disorder in children and adolescents. Am J Psychiatry. 2000;157(8):1229-35.

53. Simons M, Herpertz-Dahlmann B. Traumata und Traumafolgestörungen bei Kindern und Jugendlichen-eine kritische Übersicht zu Klassifikation und diagnostischen Kriterien. Z Kinder Jugendpsychiatr Psychother. 2008;36(3):151-61.

54. Tangney JP, Wagner P, Fletcher C, Gramzow R. Shamed into anger? The relation of shame and guilt to anger and selfreported aggression. J Pers Soc Psychol. 1992;62(4):669-75.

55. Völkl-Kernstock S, Huemer J, Jandl-Jager E, AbensbergTraun M, Marecek S, Pellegrini E, et al. Experiences of domestic and school violence among child and adolescent psychiatric outpatients. Child Psychiatry Hum Dev. 2015; 1-5. doi:10.1007/s10578-015-0602-7.

56. Weierich MR, Nock MK. Posttraumatic stress symptoms mediate the relation between childhood sexual abuse and nonsuicidal self-injury. J Consult Clin Psychol. 2008;76(1):39-44.

57. Wittchen HU, Wunderlich U, Gruschwitz S, Zaudig M. SKID-I. Strukturiertes Klinisches Interview für DSM-IV. Achse I: Psychische Störungen (SCID-I. Structured Clinical Interview for DSM-IV, Axis I Disorders). Göttingen: Hogrefe; 1997.

58. Wöller W. Trauma und Persönlichkeitsstörungen: Psychodynamisch-integrative Therapie. Stuttgart: Schattauer; 2006.

59. Youngstrom E, Löber R, Stouthamer-Löber M. Patterns and correlates of agreement between parent, teacher, and male adolescent ratings of externalizing and internalizing problems. JConsult Clin Psychol. 2000;68(6):1038. 